Interfaces and Free Boundaries 15 (2013), 121-140

DOI 10.4171/IFB/297

\title{
Eikonal equations on ramified spaces
}

\author{
FABIO CAMILLI \\ Dipartimento di Scienze di Base e Applicate per l'Ingegneria, "Sapienza” Università di Roma, \\ 00161 Roma, Italy \\ E-mail: camilli@dmmm.uniroma1.it \\ DIRK SCHIEBORN \\ Eberhard-Karls University, Tübingen, Germany \\ E-mail:dirk@schieborn.de \\ Claudio MARCHI \\ Dip. di Matematica, Università di Padova, via Trieste 63, 35121 Padova, Italy \\ E-mail:marchi@math.unipd.it
}

[Received 25 January 2012 and in revised form 29 January 2013]

\begin{abstract}
We generalize the results in [23] to higher dimensional ramified spaces. For this purpose we introduce ramified manifolds and, as special cases, locally elementary polygonal ramified spaces (LEP spaces). On LEP spaces we develop a theory of viscosity solutions for Hamilton-Jacobi equations, providing existence and uniqueness results.
\end{abstract}

2010 Mathematics Subject Classification: Primary 49L25; Secondary 58G20, 35F20

Keywords: Hamilton-Jacobi equation; ramified space; viscosity solution; comparison principle.

\section{Introduction}

In [22], [23] a theory of viscosity solution for Hamilton-Jacobi equations of eikonal type on topological networks was developed providing existence, uniqueness and stability results. In this paper we generalize these results to higher dimensional ramified spaces.

In literature, many different ways of introducing ramified spaces (cf. [18], [20], [21]) or branched manifolds (cf. [25]) are available. The definitions vary in different aspects, depending on the kind of theory to be developed. In a general approach, subsets of classic differentiable manifolds are glued together along parts of their boundaries by means of the topological gluing operation. Another, more specific, definition requires the uniqueness of the tangent space at ramification points (cf. [25]) by describing how the branches should be situated relatively to each other in the ambient space.

Here we choose an approach which is very similar to the concept of a manifold with boundary. The basic idea is that, in contrast to classic topological manifolds, besides points at which it is locally homeomorphic to an Euclidean space (simple points), a ramified topological manifold may also contain ramification points at which it is locally homeomorphic to some kind of "Euclidean ramified space". The latter, called elementary ramified space, can be visualized as a collection of closed Euclidean half spaces glued together at their boundary hyperplanes. Consequently, small neighborhoods of a given ramification point split up into different branches corresponding to the branches of the homeomorphic elementary ramified space. If we endow these ramified topological manifolds with suitable differentiable structures, then we end up with an extension of the concept 
of tangent space at ramification points. This generalization should have the property that a real function defined in a neighborhood of a ramification point can be differentiated in the direction of each branch (of course, incident at the ramification point). In other words, each branch contributes a different tangent space.

Once we have introduced the differentiable structure on ramified spaces, we will see that for each of the branches emanating from a fixed ramification point $x$, a normal direction at $x$ on this branch is well-defined. The possibility to differentiate in the normal directions at ramification points is crucial for our theory, as it will turn out that a general definition of viscosity solutions on ramified manifolds depends on this very possibility. In fact, the notion of viscosity solutions introduced in [22], [23] differs from its classical one by the transition conditions we have additionally imposed at ramification points. The concept of $(j, k)$-test functions (see definition 3.7) allows us to ignore the ramification set by treating two branches as a single connected one; indeed, the $(j, k)$ differentiability links the two derivatives of a function with respect to a given pair of branches which are incident at the same ramification point. It suggests itself to apply this pattern in case of manifolds of dimension $n$ which have a certain manifold of dimension $n-1$ in common, as long as this manifold is smooth enough to ensure that we have well-defined normal derivatives with respect to each incident branch manifold.

In order not to get lost in too general approaches, we restrict ourselves to a rather simple, but still sufficiently general, class of ramified manifolds, the so-called locally elementary polygonal ramified spaces (briefly, LEP spaces), which are characterized by two main criteria: on the one hand, LEP spaces are ramified spaces in the sense of Lumer [18] (see Definition 2.1) meeting the additional requirement that each branch is a flat $n$-dimensional submanifold of $\mathbb{R}^{n+1}$. On the other hand, they are ramified manifolds in the sense described above. Hence they can be visualized as polygonal subsets of hyperplanes in $\mathbb{R}^{n+1}$ which are glued together along certain edges, with the restriction that corner points cannot occur. The term "locally elementary" refers to the fact that they are locally homeomorphic to an open subset either of a $n$-dimensional Euclidean space or of an elementary ramified space. Once the notion of viscosity solutions has been correctly extended to LEP spaces, the development of the theory follows the line of the one devised for topological networks in [23]. Consequently we prove a comparison principle giving the uniqueness of the continuous viscosity solution. Moreover we show existence of the viscosity solution via an adaptation of the Perron's method and we also provide a representation formula for the solution of the Dirichlet problem.

We mention that Hamilton-Jacobi equation and viscosity solutions on differentiable manifolds have been studied in [3], [19]. The theory of linear and semilinear differential equations on nonsmooth manifolds such as ramified spaces has been developed, since the seminal paper [18], in a large extent [16] and it is currently an active field of research ( [11], [13], [17]). For fully nonlinear equations such as Hamilton-Jacobi equations, the theory is at the beginning and, besides [23] and the companion paper [7], different approaches have been pursued for the case of networks in the recent papers [1] and [15] and for stratified domains in [6]. The present paper can been seen as a first attempt to extend the theory of viscosity solutions for eikonal equations to general ramified spaces.

Finally, let us recall that Hamilton-Jacobi equations of eikonal type play an important role in several fields as geometric optics, homogenization, singular perturbation, weak KAM theory, granular matter theory, etc. (for instance, see [2], [8], [9], [10] and references therein). For example, if the Hamiltonian is positive homogeneous, the corresponding Hamilton-Jacobi equation is of geometric type and it is connected with flame propagation models and evolution of curves with speed of propagation depending on the normal direction (see [12], [24] and reference therein). The 
results of this paper are therefore of interest in extending the theory of front propagation developed for the Euclidean space and smooth manifolds to more general ambient spaces such as polyhedra and ramified spaces. Other applications of our theory concern computation of minimal paths on polyhedra, optimal control problems and differential games with discontinuous trajectories across a hyper-surface, granular matter theory on tent structures (we refer the reader to [6], [5] for optimal control problems with other irregular structures).

The paper is organized as follows. In Section 2 we introduce the definition and give various examples of ramified spaces. In Section 3 we study the differential structure of a ramified space. Section 4 is devoted to the notion of viscosity solution, while in Section 5 and 6 we prove uniqueness and, respectively, existence of a viscosity solution. In Section 7 we consider the Dirichlet problem and we obtain a representation formula for its solution.

\section{Ramified spaces}

In this section we introduce the geometric objects we will study in this paper. The general definition of ramified space is due to Lumer [18].

DEFINITION 2.1 Let $R^{\star}$ be a non-empty, locally compact space with a countable basis. Let $\mathcal{L}=$ $\left\{R_{j}\right\}_{j \in J}$ be a countable family of non empty open subsets $R_{j}$ of $R^{\star}$ and let $N_{E}^{\star}$ be a closed, possible empty, subset of $N^{\star}:=R^{\star} \backslash \cup_{j \in J} R_{j}$ with the property that it contains each point of $N^{\star}$ which is contained in the boundary of exactly one $R_{j}$. Then $R:=R^{\star} \backslash N_{E}^{\star}$ is a ramified space (induced by $\left.\left(R^{\star}, \mathcal{L}, N_{E}^{\star}\right)\right)$ if

- $\bar{R}_{j} \cap \bar{R}_{k} \subset \partial \bar{R}_{j} \cap \partial \bar{R}_{k}$ for all $j \neq k, j, k \in J$,

- $R^{\star}=\cup_{j \in J} \bar{R}_{j}$,

- $\left\{R_{j}\right\}_{j \in J}$ is locally finite in $R^{\star}$,

- $R$ is connected.

The set $\partial R=N_{E}^{\star}$ is called the boundary of $R$ while the set $N_{R}=N^{\star} \backslash N_{E}^{\star}$ the ramification space of $R$. We set $\partial_{R} R_{j}:=\partial R_{j} \cap N_{R}$ and $\tilde{R}_{j}:=R_{j} \cup \partial_{R} R_{j}$.

We also consider polygonal ramified space.

DEFINITION 2.2 A ramified space $R$ is said a $n$-dimensional polygonal ramified space if - $R^{\star} \subset \mathbb{R}^{n+1}$ with the endowed topology,

- For each $j \in J$, there is a hyperplane $P_{j} \subset \mathbb{R}^{n+1}$ such that $R_{j}=\Omega_{j}$ is a bounded subset of $P_{j}$, - All $P_{j}, j \in J$, are pairwise distinct.

EXAMPLE 2.1 A topological network is a collection of pairwise different points in $\mathbb{R}^{n}$ connected by continuous, non self-intersecting curves. More precisely (see [23]), let $V=\left\{v_{i}, i \in I\right\}$ be a finite collection of pairwise different points in $\mathbb{R}^{n}$ and let $\left\{\pi_{j}, j \in J\right\}$ be a finite collection of continuous, non self-intersecting curves in $\mathbb{R}^{n}$ given by $\pi_{j}:\left[0, l_{j}\right] \rightarrow \mathbb{R}^{n}, l_{j}>0, j \in J$. Defined $e_{j}:=\pi_{j}\left(\left(0, l_{j}\right)\right), \bar{e}_{j}:=\pi_{j}\left(\left[0, l_{j}\right]\right)$ and $E:=\left\{e_{j}: j \in J\right\}$, assume that

(i) $\pi_{j}(0), \pi_{j}\left(l_{j}\right) \in V$ for all $j \in J$,

(ii) $\#\left(\bar{e}_{j} \cap V\right)=2$ for all $j \in J$,

(iii) $\bar{e}_{j} \cap \bar{e}_{k} \subset V$, and $\#\left(\bar{e}_{j} \cap \bar{e}_{k}\right) \leqslant 1$ for all $j, k \in J, j \neq k$.

(iv) For all $v, w \in V$ there is a path with end-points $v$ and $w$ (i.e. a sequence of edges $\left\{e_{j}\right\}_{j=1}^{N}$ such that $\#\left(\bar{e}_{j} \cap \bar{e}_{j+1}\right)=1$ and $\left.v \in \bar{e}_{1}, w \in \bar{e}_{N}\right)$.

Then $\bar{\Gamma}:=\bigcup_{j \in J} \bar{e}_{j} \subset \mathbb{R}^{n}$ is called a (finite) topological network in $\mathbb{R}^{n}$.

A topological network is a ramified space with $R^{\star}=V \cup E, R_{j}=e_{j}, N^{\star}=V, N_{E}^{\star}$ any subset 
of $V$ containing all the vertices with only one incident edge and $N_{R}=N^{\star} \backslash N_{E}^{\star}$.

EXAMPLE 2.2 Let $\Gamma$ be a topological network as in Example 2.1. For $v \in V$, set $\operatorname{deg}(v)$ the number of the $\operatorname{arcs} e_{j}, j \in J$, incident at the vertex $v$ and define $\tilde{\Gamma}=\Gamma \backslash\{v \in V: \operatorname{deg}(v)=1\}$. Then for $n \geqslant 2$, the set $M:=\tilde{\Gamma} \times \mathbb{R}^{n-1}$ is called a $n$-dimensional topological network ( [21]). In this case $R^{\star}=(V \cup E) \times \mathbb{R}^{n-1}, R_{j}=e_{j} \times \mathbb{R}^{n-1}, N^{\star}=V \times \mathbb{R}^{n-1}, N_{E}^{\star}$ any subset of $N^{\star}$ containing the set $\cup_{\{v: \operatorname{deg}(v)=1\}}\left(v \times \mathbb{R}^{n-1}\right)$.

If the edges $\left\{e_{j}\right\}_{j \in J}$ of a topological network $\Gamma$ are segments, then $\Gamma$ and the corresponding $n$-dimensional topological networks defined as in Example 2.2 are polygonal ramified spaces in the sense of Definition 2.2. See figure 1.

EXAMPLE 2.3 Let $\Omega^{\star}$ be the surface of the $(n+1)$-dimensional cube $C^{n+1} \subset \mathbb{R}^{n+1}$ and let $\Omega_{j}$, $j=1, \ldots, 2(n+1)$, be its open faces. Furthermore let $\partial \Omega:=N_{E}^{\star}$ be any closed (possible empty) subset of the union of the edges of the cube with the property that $\Omega=\Omega^{\star} \backslash N_{E}^{\star}$ is connected. Then $\Omega:=\Omega^{\star} \backslash N_{E}^{\star}$ is a polygonal ramified space.

An important example of ramified space is the elementary ramified space, since it is the space of the parameters for LEP spaces and ramified manifolds we will define in the following.

DEFINITION 2.3 Given $n \geqslant 1$ and $r \geqslant 2$, a $n$-dimensional elementary ramified space of order $r$, denoted by $\mathbb{R}_{r}^{n}$, is the union of $r$ half spaces $\mathbb{R}_{r, j}^{n}, j \in\{1, \ldots, r\}$, of dimension $n$ which are included in $\mathbb{R}^{n+1}$ and have $\mathbb{R}^{n-1}$ in common.

If we set $\mathbb{R}_{\geqslant 0}^{n}=\left\{\left(x_{1}, x^{\prime}\right) \in \mathbb{R} \times \mathbb{R}^{n-1}: x_{1} \geqslant 0\right\}$, then we can identify $\mathbb{R}_{r}^{n}$ and $\mathbb{R}_{r, j}^{n}$ with

$$
\begin{aligned}
& \mathbb{R}_{r}^{n}=\left(\mathbb{R}_{\geqslant 0}^{n} \times\{1, \ldots, r\}\right) / \Re \\
& \mathbb{R}_{r, j}^{n}=\left\{(x, j): x \in \mathbb{R}_{\geqslant 0}^{n}\right\}
\end{aligned}
$$

where $\Re$ is the equivalence relation which for each choice of $x^{\prime} \in \mathbb{R}^{n-1}$ identifies the points $\left(\left(0, x^{\prime}\right), j\right) \in \mathbb{R}_{r, j}^{n}$ for $j \in\{1, \ldots, r\}$. The set $\mathbb{R}_{r, j}^{n}$ is said the (closed) $j$-branch of $\mathbb{R}_{r}^{n}$ while the set

$$
\Sigma_{r}^{n}=\left\{\left(\left(0, x^{\prime}\right), j\right): x^{\prime} \in \mathbb{R}^{n-1}, j \in\{1, \ldots, r\}\right\}
$$

is called the ramification space of $\mathbb{R}_{r}^{n}$.

Endowed with the topology induced by the path distance, $\mathbb{R}_{r}^{n}$ is a connected, separable, locally compact topological space. Observe that $\mathbb{R}_{r}^{n}$ can be identified with $\mathbb{R}^{n}$ if $r=2$.

In order to give the definition of ramified manifolds, we need to introduce the notion of diffeomorphism on $\mathbb{R}_{r}^{n}$.

Definition 2.4 (1) Let $U \subset \mathbb{R}_{r}^{n}$ be an open set and $f: U \rightarrow \mathbb{R}^{m}$. Then, for $0 \leqslant l \leqslant \infty, f$ is said $C^{l}$-differentiable at $x \in U$ if the following holds:

(i) If $x \in \mathbb{R}_{r, j}^{n} \backslash \Sigma_{r}^{n}$, for some $j \in\{1, \ldots, r\}$, then $f$ is $l$ times continuously differentiable at $x$ in the standard sense.

(ii) If $x \in \Sigma_{r}^{n}$, then for each $j \in\{1, \ldots, r\}$, there is a domain $V_{j} \subset \mathbb{R}^{n}$ and $f_{j} \in C^{l}\left(V_{j}, \mathbb{R}^{m}\right)$ such that $x \in V_{j}$ and $f_{j} \equiv f$ on $V_{j} \cap \mathbb{R}_{r, j}^{n}$ (having identified $\mathbb{R}_{r, j}^{n}$ with $\mathbb{R}_{\geqslant 0}^{n}$ ).

(2) Let $U, V \subset \mathbb{R}_{r}^{n}$ be open sets and $\varphi: U \rightarrow V$ an homeomorphism. Then $\varphi$ is said a diffeomorphism if for all $j \in\{1, \ldots, r\}$ the respective restrictions of $\varphi$ and $\varphi^{-1}$ to $\mathbb{R}_{r, j}^{n} \cap U$ and to $R_{r, j}^{n} \cap V$ are $C^{\infty}$-differentiable. 
We are now ready to give the definition of topological ramified manifold and differentiable ramified manifold.

DEFINITION 2.5 A set $M$ is called a $n$-dimensional topological ramified manifold if it is endowed with a Hausdorff topology and if for any $x \in M$, there is a neighborhood $U \subset M$ of $x$ such that there is an integer $r=r(x) \geqslant 2$, an open set $V \subset \mathbb{R}_{r}^{n}$ with $V \cap \Sigma_{r}^{n} \neq \emptyset$ and a homeomorphism $X: U \rightarrow V$ with $X(x) \in \Sigma_{r}^{n}$.

The number $r(x)$ is called ramification order of $x$. A point $x \in M$ is said a simple point if $r(x)=2$, a ramification point if $r(x) \geqslant 3$. The set of all ramification points is denoted by $\Sigma$ and it is called ramification space of $M$. If $x \in \Sigma$, we set Inc $x:=\left\{j \in J: x \in \partial \Omega_{j}\right\}$.

REMARK 2.1 Observe that, since $\mathbb{R}_{2}^{n}$ can be identified with $\mathbb{R}^{n}$, topological ramified manifolds are locally homeomorphic to a $n$-dimensional Euclidean space at simple points.

DEFINITION 2.6 A set $M$ is called a $n$-dimensional differentiable ramified manifold if $M$ is a $n$ dimensional topological ramified manifold and there is a family of local charts $\left\{U_{\alpha}, X_{\alpha}\right\}$, i.e. open set $U_{\alpha} \subset M$ and injective mappings $X_{\alpha}: U_{\alpha} \rightarrow \mathbb{R}_{r(\alpha)}^{n}$, with the following properties

(i) For any $\alpha, \alpha^{\prime}$ with $V=U_{\alpha} \cap U_{\alpha^{\prime}} \neq \emptyset$, the sets $X_{\alpha}(V)$ and $X_{\alpha^{\prime}}(V)$ are open in $R_{r(\alpha)}^{n}$ and $\mathbb{R}_{r\left(\alpha^{\prime}\right)}^{n}$, respectively. Moreover the map $\varphi: X_{\alpha}(V) \rightarrow X_{\alpha^{\prime}}(V)$ given by $\varphi:=X_{\alpha^{\prime}} \circ X_{\alpha}^{-1}$ is a diffeomorphism in the sense of Definition 2.4.

(ii) $\bigcup_{\alpha} U_{\alpha}=M$.

(iii) The family $\left\{U_{\alpha}, X_{\alpha}\right\}$ is maximal with respect to the conditions i) and ii).

We introduce a class of flat ramified manifolds.

DEFINITION 2.7 A $n$-dimensional polygonal ramified space $\Omega$ (see Definition 2.2) is called locally elementary if it is also a differentiable manifold. Locally elementary ramified space will be called LEP spaces in the following.

EXAMPLE 2.4 Topological networks and $n$-dimensional topological networks are topological ramified manifolds. If the maps $\left\{\pi_{j}\right\}_{j \in J}$ in the definition of $\Gamma$ are diffeomorphisms, they are also differentiable ramified manifolds. If the edges $\left\{e_{j}\right\}_{j \in J}$ of $\Gamma$ are segments, a $n$-dimensional topological network is a LEP space.

The set of ramification points $\Sigma$ is given by $\{v \in V: \operatorname{deg}(v)>1\}$ for a topological network and by $\cup_{r>2} M_{r}$ where $M_{r}=\cup_{\{v \in V: \operatorname{deg}(v)=r\}}\left(\{v\} \times \mathbb{R}^{n-1}\right)$ for a $n$-dimensional topological network. Note that $\Sigma=N_{R}$ for a LEP space.

EXAMPLE 2.5 The cube in the Example 2.3 is not locally homeomorphic to an elementary ramified space at the corner points. It is a LEP space if all the $2^{n}$ corner points are contained in $N_{E}^{\star}=\partial \Omega$.

\section{The differential structure of a ramified manifold}

In this section we extend the notion of tangent space to a differentiable ramified manifold. In fact, the interpretation of tangent vectors as equivalence classes of curves in $M$ can be easily transferred to ramification points.

Throughout this section, $M$ and $\Sigma$ stand respectively for a $n$-dimensional differentiable ramified manifold and for its ramification set. Let us now introduce some definitions regarding the differential structure of $M$. 


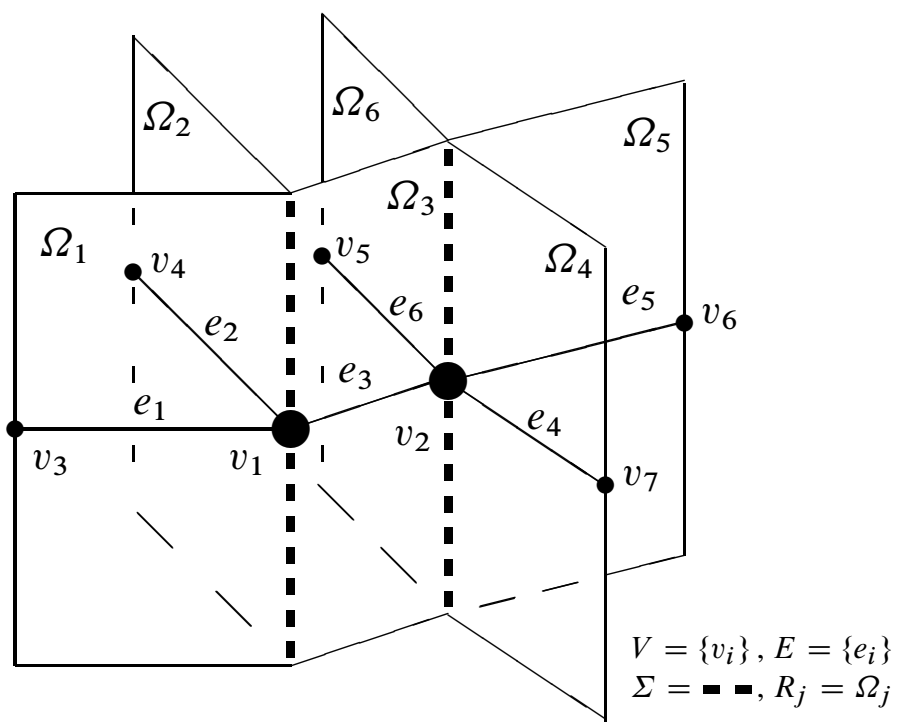

FIG. 1. Example of $n$-dimensional topological network

Definition 3.1 A continuous function $f: M \rightarrow \mathbb{R}$ is said to be $C^{l}$-differentiable at $x \in M$ if for any local chart $(U, X)$ around $x$, the function $f \circ X^{-1}$ is $C^{l}$-differentiable in sense of Definition 2.4.

DEFinition 3.2 Let $x \in \Sigma$ and $r=r(x)$. Let $\gamma:(-\epsilon, \epsilon) \rightarrow M$ with $\gamma(0)=x$ be a continuous curve and $j \in\{1, \ldots, r\}$. We say that $\gamma$ reaches $x$ from the branch $j$ whenever there exists a chart $(U, X)$ with $x \in U$ and $\delta>0$ such that

$$
\tilde{\gamma}(t)=(X \circ \gamma)(t) \in \mathbb{R}_{r, j}^{n} \quad \text { for all } t \in(-\delta, 0) .
$$

We denote by $\mathrm{C}_{j}(x)$ the set of all the curves reaching $x$ from the branch $j$ and we set

$$
\mathrm{e}(x)=\cup_{j \in J} \mathrm{e}_{j}(x) .
$$

DEFINITION 3.3 Let $x \in \Sigma, r=r(x)$ and $\gamma_{1}, \gamma_{2} \in \mathrm{C}(x)$. We say that $\gamma_{1}$ and $\gamma_{2}$ are equivalent if for all functions $f: M \rightarrow \mathbb{R}$ which are $C^{\infty}$-differentiable at $x$ we have

$$
\left(f \circ \gamma_{1}\right)_{-}^{\prime}(0)=\left(f \circ \gamma_{2}\right)_{-}^{\prime}(0)
$$

where the derivatives are left-sided. We denote the set of equivalence classes by $T_{x} M$, the tangent space of $M$ at $x$, and we say that $\xi \in T_{x} M$ is a $j$-tangent vector at $x, 1 \leqslant j \leqslant r$, if $\xi$ contains a curve reaching $x$ from the branch $j$. We set

$$
\xi(f):=(f \circ \gamma)_{-}^{\prime}(0), \quad \gamma \in \xi .
$$

The set of all $j$-tangent vectors at $x$ ( $j$-tangent space at $x$ ) is denoted by $T_{x}^{j} M$. The set $T_{x} \Sigma:=$ $\cap_{j} T_{x}^{j} M$ is called the $\Sigma$-tangent space at $x$ and any $\xi \in T_{x} \Sigma$ is said a $\Sigma$-tangent vector at $x$. 
REMARK 3.1 If $x \in \Sigma$, the tangent space $T_{x} M$ is not a vector space. Instead it can be identified with an elementary ramified space $\mathbb{R}_{r}^{n}$, where

$$
R_{r, j}^{n}=T_{x}^{j} M, 1 \leqslant j \leqslant r, \quad \Sigma_{r}^{n}=T_{x} \Sigma .
$$

Hence $T_{x} \Sigma \subset T_{x}^{j} M$ can be identified with $\mathbb{R}^{n-1}$ and $T_{x}^{j} M$ with $\mathbb{R}_{\geqslant 0}^{n}$.

Definition 3.4 Let $1 \leqslant j \leqslant r, f: M \rightarrow \mathbb{R}$ continuously differentiable at $x$ (see Def. 3.1) and $\xi_{1}, \ldots, \xi_{n}$ a basis of $T_{x}^{j} M$. We define the $j$-gradient $D^{j} f \in T_{x}^{j} M$ of $f$ at $x$ by

$$
D^{j} f(x):=\sum_{i=1}^{n} \xi_{i}(f) \xi_{i}
$$

We consider the case of an elementary ramified space and we introduce some notations for the derivatives at the ramification set.

DEFINITION 3.5 Let $r \geqslant 3$ and let $x \in \Sigma_{r}^{n} \subset \mathbb{R}_{r}^{n}$. Let $u: \mathbb{R}_{r}^{n} \rightarrow \mathbb{R}$ be continuously differentiable at $x$. We denote by $\partial_{1} u(x), \ldots, \partial_{n-1} u(x)$ the directional derivatives of $u$ at $x$ with respect to the canonical basis $e_{1}, \ldots, e_{n-1}$ of $\Sigma_{r}^{n} \equiv \mathbb{R}^{n-1}$. For $1 \leqslant j \leqslant r$ we denote by $\partial_{\nu_{j}} u(x)$ the directional derivative of $u$ at $x$ with respect to the inward unit normal $v_{j}$ of $\mathbb{R}_{r, j}^{n} \equiv \mathbb{R}_{\geqslant 0}^{n}$ at $x$. See Figure 2 .

We now restrict our attention to LEP spaces, which have the important property that around any given point we can always choose a chart induced by the canonical identification with the Euclidean space $\mathbb{R}^{n}$ or a suitable elementary ramified space. This is stated in the following proposition.

PROPOSITION 3.1 Let $\Omega$ be a LEP space and $\Sigma$ its ramification set. For any $x \in \Omega$, there is a neighborhood $V_{x}$ of $x$ and a canonical identification $\mathfrak{i}_{x}: V_{x} \rightarrow \mathfrak{i}_{x}\left(V_{x}\right)$ where $\mathfrak{i}_{x}(x)=0$ and

(i) if $x \notin \Sigma$ then $\mathfrak{i}_{x}\left(V_{x}\right) \subset \mathbb{R}^{n}$,

(ii) if $x \in \Sigma$ then $\mathfrak{i}_{x}\left(V_{x}\right) \subset \mathbb{R}_{r(x)}^{n}$ with $\mathfrak{i}_{x}\left(V_{x} \cap \Sigma\right) \subset \Sigma_{r(x)}^{n}$.

In the latter case, $\mathfrak{i}_{x}$ induces a bijective map $\ell_{x}$ between the index set Inc $x_{x}$ and the set $\{1, \ldots, r(x)\}$.

We now consider derivatives of a function on a LEP space at ramification points. For any $x \in \Omega$, we always fix a canonical identification chart $\left(V_{x}, \mathfrak{i}_{x}\right)$ as defined in Proposition 3.1 and all the concepts will be expressed in terms of the chart $\mathfrak{i}_{x}$ for sake of simplicity. However it is easy to verify that they in fact do not depend on the choice of specific chart.

DEFINITION 3.6 Let $\Omega$ be a LEP space with ramification set $\Sigma$. Let $x \in \Sigma, r=r(x)$. Let $V \subset \Omega$ be a neighborhood of $x$ and let $u: V \rightarrow \mathbb{R}$ be a function which is continuously differentiable at $x$. Following the notation of Definition 3.5, we set

$$
\begin{aligned}
\partial_{i} u(x) & :=\partial_{i}\left(u \circ \mathfrak{i}_{x}^{-1}\right)(0), & i=1, \ldots, n-1 \\
\partial_{\nu_{j}} u(x) & :=\partial_{\nu_{\ell_{x}(j)}}\left(u \circ \mathfrak{i}_{x}^{-1}\right)(0), & j \in \operatorname{Inc}_{x} \\
\partial^{j} u(x) & :=\left(\partial_{1} u(x), \ldots, \partial_{n-1} u(x), \partial_{\nu_{j}} u(x)\right) &
\end{aligned}
$$

where $\ell_{x}$ is defined as in Proposition 3.1 (note that for each $j \in \operatorname{Inc}_{x}$ the collection $\left\{\partial_{1}, \ldots \partial_{n-1}, \partial_{v_{j}}\right\}$ forms a basis of $T_{x}^{j} \Omega$ ).

For any function $u: \Omega \rightarrow \mathbb{R}$ and each $j \in J$ we denote by $u^{j}: \bar{\Omega}_{j} \rightarrow \mathbb{R}$ the restriction of $u$ to $\bar{\Omega}_{j}$, i.e.,

$$
u^{j}(x):=u \circ \mathfrak{i}_{x}^{-1}(0) \quad \text { for } x \in \bar{\Omega}_{j} .
$$




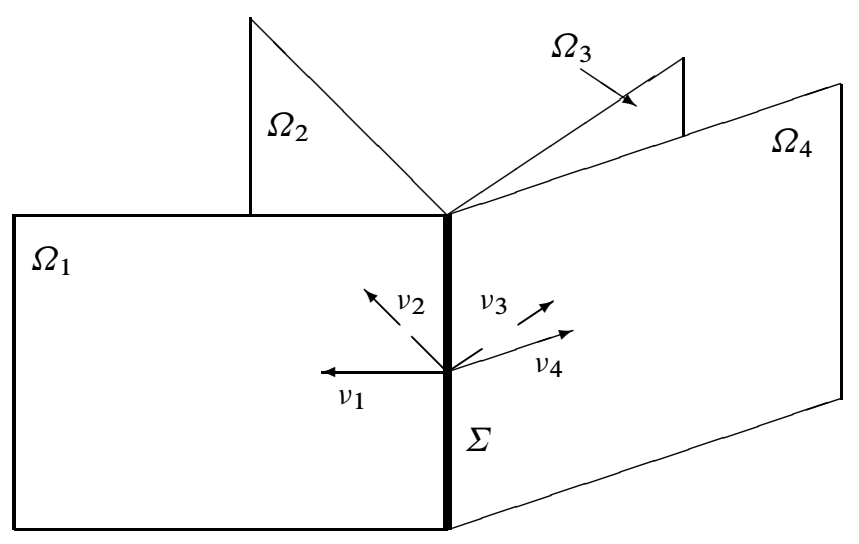

FIG. 2. LEP space with normals at a ramification point

We denote by $C(\Omega)$ the space of continuous function on $\Omega$. This in particular implies that $u^{j} \in$ $C\left(\bar{\Omega}_{j}\right)$ and

$$
u^{j}(x)=u^{k}(x) \text { for any } x \in \Sigma, j, k \in \operatorname{In} c_{x} .
$$

In a similar way we define the space of upper semi-continuous functions $\operatorname{USC}(\Omega)$ and the space of lower semi-continuous functions $\operatorname{LSC}(\Omega)$.

In [23], we introduced the concept of $(j, k)$-test function on a topological network $\Gamma$, treating two edges $l_{j}$ and $l_{k}$ incident at a vertex $v_{i}$ as one connected edge and imposing that the derivatives in the direction of the incident edges, taking into account their orientations, coincide at $v_{i}$. In other terms, a test function, considered as a function defined on $e_{j} \cup e_{k}$, is differentiable at the interior point $v_{i}$.

Here we follow a similar idea for LEP spaces, linking the two normal derivatives of a test function for a given couple of branch manifolds incident at a point $x \in \Sigma$.

Definition 3.7 Let $\varphi \in C(\Omega), x \in \Sigma, k, l \in \operatorname{Inc}_{x}, k \neq l$. Then $\varphi$ is said to be $(k, l)$ differentiable at $x$ if $\varphi$ is $C^{1}$-differentiable at $x$ and if we have

$$
\partial_{v_{k}} \varphi(x)+\partial_{v_{l}} \varphi(x)=0 .
$$

Definition 3.8 Let $u: \Omega \rightarrow \mathbb{R}$ and let $\varphi$ be $C(\Omega)$.

- Let $j \in J$ and let $x \in \Omega_{j}$. We say that $\varphi$ is an upper (lower) test function of $u$ at $x$ if $\varphi$ is $C^{1}$-differentiable at $x$ and $u-\varphi$ attains a local maximum (minimum) at $x$.

- Let $x \in \Sigma$ and $k, l \in \operatorname{Inc}_{x}, k \neq l$. We say that $\varphi$ is a $(k, l)$ upper (lower) test function of $u$ at $x$ if $\varphi$ is $(k, l)$-differentiable at $x$ and $u-\varphi$ attains a local maximum (minimum) at $x$ with respect to $\Omega_{k, l}:=\bar{\Omega}_{k} \cup \bar{\Omega}_{l}$.

\section{Viscosity solutions}

Since now on, $\Omega$ and $\Sigma$ stand respectively for a LEP space and for its ramification set. We introduce the class of Hamilton-Jacobi equations of eikonal type we consider in this paper. An Hamiltonian 
$H=\left(H^{j}\right)_{j \in J}$ is a family of mappings $H^{j}: \tilde{\Omega}_{j} \times T_{x}^{j} \Omega \rightarrow \mathbb{R}$ (recall: $\partial_{R} \Omega_{j}:=\partial \Omega_{j} \cap \Sigma$, $\tilde{\Omega}_{j}=\Omega_{j} \cup \partial_{R} \Omega_{J}$ ). By means of the canonical identification map $\mathfrak{i}_{x}$ around a fixed point $x \in \tilde{\Omega}_{j}$ we can think of $H^{j}$ as a mapping $\tilde{H}^{j}: V \times \mathbb{R}^{n} \rightarrow \mathbb{R}$ defined by the identification

$$
H^{j}\left(y, D^{j} u(y)\right)=\tilde{H}^{j}\left(\mathfrak{i}_{x}(y), \partial^{j} u(y)\right) \quad \forall y \in V_{x}
$$

(see (3.2) and (3.3)) where $V$ is a neighborhood of $0 \in \mathbb{R}^{n}$ or $0 \in \mathbb{R}_{\geqslant 0}^{n}$ provided that $x \in \Omega_{j}$ or $x \in \Sigma$, respectively. In the sequel we will speak of $H^{j}$ under the canonical identification (around $x$ ) whenever we refer to $H^{j}$ in the sense of (4.1). We assume the Hamiltonian $H=\left(H^{j}\right)_{j \in J}$ fulfills the following properties

$$
\begin{aligned}
& H^{j} \text { is continuous on } \tilde{\Omega}_{j} \times \mathbb{R} ; \\
& H^{j}(x, p) \rightarrow+\infty \quad \text { as }|p| \rightarrow \infty \text { for } x \in \tilde{\Omega}_{j} ; \\
& \text { for each } x \in \Sigma, j \in \operatorname{Inc}_{x}, p_{n} \mapsto H^{j}\left(x,\left(p_{1}, \ldots, p_{n-1}, \cdot\right)\right) \text { is not decreasing for } p_{n} \geqslant 0 ; \\
& \text { for each } x \in \Sigma, j, k \in \operatorname{Inc}_{x}, H^{j}(x, p)=H^{k}(x, p) \quad \forall p \in \mathbb{R}^{n} ; \\
& \text { for each } x \in \Sigma, j \in \operatorname{Inc}_{x}, H^{j}\left(x,\left(p^{\prime}, p_{n}\right)\right)=H^{j}\left(x,\left(p^{\prime},-p_{n}\right)\right) \quad \forall p^{\prime} \in \mathbb{R}^{n-1}, p_{n} \in \mathbb{R} .
\end{aligned}
$$

REMARK 4.1 Assumptions (4.2)-(4.3) are standard conditions in viscosity solution theory (see f.e. [14]) to ensure existence and uniqueness of the solution. Assumptions (4.5) and (4.6) represent compatibility conditions across the ramification set; the former guarantees a continuity condition at $x \in \Sigma$ for the Hamiltonians defined on two different branches while the latter states the invariance with respect to orientation of the inward normal $v_{j}$. Under hypotheses (4.3) and (4.6), assumption (4.4) is fulfilled provided that, for $x \in \Sigma, H^{j}(x, \cdot)$ is convex. Observe that thanks to the identification (4.1), (4.4)-(4.6) induce corresponding properties for the Hamiltonian $\tilde{H}=$ $\left\{\tilde{H}^{j}\right\}_{j \in J}$.

EXAMPLE 4.1 A typical example of Hamiltonian satisfying the previous assumptions is given by the family $H^{j}(x, p)=|p|^{2}-f^{j}(x)$ where the functions $f^{j}: \bar{\Omega}_{j} \rightarrow \mathbb{R}$ are continuous, non negative and satisfies the compatibility condition $f^{j}(x)=f^{k}(x)$ if $x \in \partial_{R} \Omega_{j} \cap \partial_{R} \Omega_{k}$.

We introduce the definition of viscosity solution for the Hamilton-Jacobi equation of eikonal type

$$
H(x, D u)=0, \quad x \in \Omega .
$$

For $x \in \Sigma$, we define by $\pi_{j}: T_{x}^{j} \Omega \rightarrow T_{x} \Sigma$ the projection on the tangent space of $\Sigma$, i.e.

$$
\pi_{j}(p):=\sum_{m=1}^{n-1} p_{m} \partial_{m} \quad \text { for } \quad p=p_{n} \partial_{v_{j}}+\sum_{m=1}^{n-1} p_{m} \partial_{m} .
$$

Definition 4.1 A function $u \in \operatorname{USC}(\Omega)$ is called a (viscosity) subsolution of (4.7) in $\Omega$ if the following holds:

(i) For any $x \in \Omega_{j}, j \in J$, and for any upper test function $\varphi$ of $u$ at $x$ we have

$$
H^{j}\left(x, D^{j} \varphi(x)\right) \leqslant 0 .
$$

(ii) For any $x \in \Sigma$, for any $j, k \in \operatorname{Inc}_{x}$ and for any $(j, k)$ upper test function $\varphi$ of $u$ at $x$ we have

$$
H^{j}\left(x, D^{j} \varphi(x)\right) \leqslant 0 .
$$


A function $u \in \operatorname{LSC}(\Omega)$ is called a (viscosity) supersolution of (4.7) in $\Omega$ if the following holds:

(i) For any $x \in \Omega_{j}, j \in J$, and for any lower test function $\varphi$ of $u$ at $x$ we have

$$
H^{j}\left(x, D^{j} \varphi(x)\right) \geqslant 0 .
$$

(ii) For any $x \in \Sigma, p^{\prime} \in T_{x} \Sigma$ (see definition 3.3) and $j \in \operatorname{Inc}_{x}$, there exists $k \in \operatorname{Inc}_{x}, k \neq j$ such that

$$
H^{j}\left(x, D^{j} \varphi(x)\right) \geqslant 0
$$

for any $(j, k)$ lower test function $\varphi$ of $u$ at $x$ satisfying $\pi_{j}\left(D^{j} \varphi(x)\right)=p^{\prime}$.

A function $u \in C(\Omega)$ is called a (viscosity) solution of (4.7) if it is both a viscosity subsolution and a viscosity supersolution.

REMARK 4.2 (i) Near $x \in \Sigma$ the set $\Omega_{k} \cup \Omega_{j}$ is locally diffeomorphic to $\mathbb{R}^{n}$ with a tangent space given by $T_{x}^{j} \Omega \cup T_{x}^{k} \Omega$. The tangent space is composed by vectors $\left(p^{\prime}, p_{n}\right)$ with $p^{\prime} \in T_{x} \Sigma \subset$ $T_{x}^{j} \Omega \cap T_{x}^{k} \Omega$ and $p_{n} \perp T_{x} \Sigma$. Taking into account the condition of differentiability (3.4), we see that if $\varphi$ is a $(j, k)$-test function of $u$ at $x \in \Sigma$, then by (4.5)-(4.6),

$$
H^{j}\left(x, D^{j} \varphi(x)\right)=H^{k}\left(x, D^{k} \varphi(x)\right) .
$$

In other terms, at a ramification point, the equation (4.7) can be equivalently replaced by one of the two couples $\left(H^{j}(x, p), T_{x}^{j} \Omega\right)$ and $\left(H^{k}(x, p), T_{x}^{k} \Omega\right)$.

(ii) The definitions of subsolution and supersolution are not symmetric at a ramification point. It turns out that solutions of eikonal equations are distance-like functions from the boundary (see Section 7); this definition of supersolution reflects the idea that these functions have to be solutions of (4.7). Since there is always a shortest path from a ramification point to the boundary, for any branch $\Omega_{j}$ and for any $x \in \Sigma \cap \Omega_{j}$ we can connect the $x$ to the boundary contained in the branch $\Omega_{k}$ by a shortest path. Then $(j, k)$-lower test functions satisfies the definition of supersolution.

It is also worth to observe that, for $r(x)=2$, our definition of solution (resp., sub- or supersolution) coincides with the standard notion of viscosity solution (resp., sub- or supersolution).

REMARK 4.3 The definition of viscosity solution is not affected by the fact that $x$ belongs or not to the ramification set $\Sigma$. At ramification points, it requires a suitable class of test functions (different from the classical one) with appropriate differentiability properties. In this respect, our approach is different from the one in $[1,6,15]$ which require to suitably redefine the Hamiltonian at ramification points.

\section{A comparison result}

This section is devoted to the proof of a comparison theorem for (4.7). The coerciveness of the Hamiltonian (stated in (4.3)) implies the Lipschitz-continuity of subsolutions; such a regularity will be exploited in the comparison theorem.

LEMMA 5.1 Let $K$ be a compact subset of $\Omega$ and let $u$ be a subsolution of (4.7). Then there exists a constant $C_{K}$ depending only on $K$ such that

$$
|u(x)-u(y)| \leqslant C_{K} d(x, y), \quad \forall x, y \in K .
$$

The proof is standard in viscosity theory and we refer the reader to [4, Prop.II.4.1]. 
THEOREM 5.1 Let $v \in \operatorname{LSC}(\bar{\Omega})$ and $u \in U S C(\bar{\Omega})$ be respectively a supersolution to (4.7) and a subsolution of

$$
H(x, D u)=f(x), \quad x \in \Omega,
$$

with $f \in C(\Omega)$ and $f(x)<0$ for all $x \in \Omega$. If $u \leqslant v$ on $\partial \Omega$, then $u \leqslant v$ in $\bar{\Omega}$.

Proof. Assume by contradiction that there exists $z \in \Omega$ such that

$$
u(z)-v(z)=\max _{\bar{\Omega}}\{u-v\}=\delta>0 .
$$

For $\epsilon>0$ define $\Phi_{\epsilon}: \bar{\Omega} \times \bar{\Omega} \rightarrow \mathbb{R}$ by

$$
\Phi_{\epsilon}(x, y):=u(x)-v(y)-\epsilon^{-1} d(x, y)^{2}
$$

where $d(x, y)$ is the geodesic distance between two points $x$ and $y$ on the set $\bar{\Omega}$. Since $\Phi_{\epsilon}$ is an upper semi-continuous function, there exists a maximum point $\left(x_{\epsilon}, y_{\epsilon}\right)$ for $\Phi_{\epsilon}$ in $\bar{\Omega}^{2}$. By $\Phi_{\epsilon}(z, z) \leqslant$ $\Phi_{\epsilon}\left(x_{\epsilon}, y_{\epsilon}\right)$ we get

$$
\epsilon^{-1} d\left(x_{\epsilon}, y_{\epsilon}\right)^{2} \leqslant u\left(x_{\epsilon}\right)-v\left(y_{\epsilon}\right)-\delta \leqslant M,
$$

for some $M \in \mathbb{R}$. Hence

$$
\lim _{\epsilon \rightarrow 0} d\left(x_{\epsilon}, y_{\epsilon}\right)=0 .
$$

It follows that there exists $x_{0} \in \bar{\Omega}$ such that $x_{\epsilon}, y_{\epsilon} \rightarrow x_{0}$. Owing to (5.3), there holds: $\delta \leqslant$ $u\left(x_{\epsilon}\right)-v\left(y_{\epsilon}\right)$; passing to the limit, we infer $0<\delta \leqslant u\left(x_{0}\right)-v\left(x_{0}\right)$ and, in particular, $x_{0} \notin \partial \Omega$. Whence, for $\epsilon$ sufficiently small, we get: $x_{\epsilon}, y_{\epsilon}, x_{0} \in \Omega$. By (5.3) and the Lipschitz continuity of $u$ (see Lemma 5.1) we get

$$
\epsilon^{-1} d\left(x_{\epsilon}, y_{\epsilon}\right)^{2} \leqslant u\left(x_{\epsilon}\right)-u\left(y_{\epsilon}\right)+u\left(y_{\epsilon}\right)-v\left(y_{\epsilon}\right)-\delta \leqslant L d\left(x_{\epsilon}, y_{\epsilon}\right)
$$

and therefore

$$
\lim _{\epsilon \rightarrow 0^{+}} \epsilon^{-1} d\left(x_{\epsilon}, y_{\epsilon}\right)=0 .
$$

Since $x_{0} \in \Omega$, there exists $r>0$ such that

$$
B_{r}\left(x_{0}\right) \cap \partial \Omega=\emptyset
$$

and for $\epsilon$ sufficiently small, $x_{\epsilon}, y_{\epsilon} \in B_{r}\left(x_{0}\right)$. Since $\Omega$ is compact and composed by a finite number of branches $\Omega_{j}$, we have

$$
\inf _{j \in J} \inf _{k: \bar{\Omega}_{j} \cap \bar{\Omega}_{k}=\emptyset} d\left(\Omega_{j}, \Omega_{k}\right)>0 .
$$

Hence, for $\epsilon$ sufficiently small, we can assume that if $x_{\epsilon} \in \Omega_{j_{\epsilon}}$ and $y_{\epsilon} \in \Omega_{k_{\epsilon}}$, then $\bar{\Omega}_{j_{\epsilon}} \cap \bar{\Omega}_{k_{\epsilon}} \neq \emptyset$.

From now on we set $x:=x_{\epsilon}, y:=y_{\epsilon}$ and we define $\varphi_{x}(\cdot):=\epsilon^{-1} d(x, \cdot)^{2}$ and $\varphi_{y}(\cdot):=$ $\epsilon^{-1} d(\cdot, y)^{2}$ and we work with the canonical identification (4.1).

Case 1. $x, y \in \tilde{\Omega}_{j}$ for some $j \in J$ : Consider $\mathfrak{i}_{x_{0}}: V_{x_{0}} \rightarrow \mathfrak{i}_{x_{0}}\left(V_{x_{0}}\right)$ and set

$$
\tilde{x}=\mathfrak{i}_{x_{0}}(x), \quad \tilde{y}=\mathfrak{i}_{x_{0}}(y) .
$$

If $x \in \Omega_{j}$, then $\varphi_{x}(\cdot)$ is differentiable at $y$, and

$$
\partial^{j} \varphi_{x}(\tilde{y})=\epsilon^{-1} d(x, y) \frac{\tilde{y}-\tilde{x}}{|\tilde{y}-\tilde{x}|} .
$$


If $x \in \Sigma$, then we have

$$
\begin{aligned}
& \partial^{j} \varphi_{x}(\tilde{y})=\epsilon^{-1} d(x, y) \frac{\tilde{y}-\tilde{x}}{|\tilde{y}-\tilde{x}|} \\
& \left(\partial_{1} \varphi_{x}(\tilde{y}), \ldots, \partial_{n-1} \varphi_{x}(\tilde{y})\right) \in T_{x} \Sigma_{r(x)}^{n} \\
& \partial_{\nu_{k}} \varphi_{x}(\tilde{y})=-\partial_{v_{j}} \varphi_{x}(\tilde{y}) \quad \text { for any } k \in \operatorname{Inc}_{x}, k \neq j .
\end{aligned}
$$

We conclude that for any $k \in \operatorname{Inc}_{x}, k \neq j, \varphi_{x}(\cdot)$ is $(j, k)$-differentiable at $y$. A corresponding property holds for $\varphi_{y}(\cdot)$.

Since $u-\varphi_{y}$ has a maximum point at $x$ and $v+\varphi_{x}$ has a minimum point at $y$, we get

$$
\begin{aligned}
& H^{j}\left(x, D^{j} \varphi_{y}(x)\right) \leqslant f(x) \\
& H^{j}\left(y,-D^{j} \varphi_{x}(y)\right) \geqslant 0 .
\end{aligned}
$$

Moreover

$$
H^{j}\left(y,-D^{j} \varphi_{x}(y)\right)=\tilde{H}^{j}\left(\tilde{y}, \epsilon^{-1} d(x, y) \frac{\tilde{y}-\tilde{x}}{|\tilde{y}-\tilde{x}|}\right)=H^{j}\left(y, D^{j} \varphi_{y}(x)\right) .
$$

Fix $R>0$ and denote by $\omega_{j}$ the modulus of continuity of $H^{j}$ with respect to $(x, p) \in \bar{\Omega}_{j} \times$ $[-R, R]^{n}$. Hence, for sufficiently small $\epsilon>0$ we have

$$
\eta \leqslant-f(x) \leqslant H^{j}\left(y, D^{j} \varphi_{y}(x)\right)-H^{j}\left(x, D^{j} \varphi_{y}(x)\right) \leqslant \omega_{j}(d(x, y))
$$

for some $\eta>0$. By (5.5) we get a contradiction for $\epsilon \rightarrow 0$.

Case 2. $x \in \Omega_{j}, y \in \Omega_{k}$ for some $j, k \in J, j \neq k$ : Consider $\mathfrak{i}_{x_{0}}: V_{x_{0}} \rightarrow \mathfrak{i}_{x_{0}}\left(V_{x_{0}}\right)$, set $r=r\left(x_{0}\right)$ (see def. 3.6) and

$$
\tilde{x}=\mathfrak{i}_{x_{0}}(x) \in \mathbb{R}_{r, \ell_{x_{0}}(j)}^{n}, \quad \tilde{y}=\mathfrak{i}_{x_{0}}(y) \in \mathbb{R}_{r, \ell_{x_{0}}(k)}^{n} .
$$

Since $x \in \Omega_{j}, y \in \Omega_{k}$, by (5.6) there exists $z \in \tilde{\Omega}_{j} \cap \tilde{\Omega}_{k} \subset \Sigma$ such that $d(x, y)=d(x, z)+$ $d(z, y)$ and, defined $\tilde{z}=\mathfrak{i}_{x_{0}}(z) \in \Sigma_{r}^{n}$, we have

$$
\begin{aligned}
& \partial^{k} \varphi_{x}(\tilde{y})=\epsilon^{-1} d(x, y) \frac{\tilde{y}-\tilde{z}}{|\tilde{y}-\tilde{z}|} \\
& \partial^{j} \varphi_{y}(\tilde{x})=\epsilon^{-1} d(x, y) \frac{\tilde{x}-\tilde{z}}{|\tilde{x}-\tilde{z}|} .
\end{aligned}
$$

Moreover

$$
\begin{aligned}
& \partial_{i} \varphi_{x}(\tilde{y})=-\partial_{i} \varphi_{y}(\tilde{x}) \quad i=1, \ldots, n-1 \\
& \partial_{\nu_{k}} \varphi_{x}(\tilde{y})=\partial_{\nu_{j}} \varphi_{y}(\tilde{x})
\end{aligned}
$$

taking into account the definition of $v_{j}, v_{k}$. As in Case $1, \varphi_{x}$ and $\varphi_{y}$ are $(j, k)$-differentiable and $u-\varphi_{y}$ has a maximum point at $x$ and $v+\varphi_{x}$ has a minimum point at $y$. Therefore

$$
\begin{aligned}
& H^{j}\left(x, D^{j} \varphi_{y}(x)\right) \leqslant f(x) \\
& H^{k}\left(y,-D^{k} \varphi_{x}(y)\right) \geqslant 0 .
\end{aligned}
$$


Fix $R>0$ and denote by $\omega$, the modulus of continuity of every $H^{l}$ with respect to $(x, p) \in$ $\bar{\Omega}_{l} \times[-R, R]^{n}$. For sufficiently small $\epsilon>0$ we have

$$
\begin{aligned}
\eta \leqslant & -f(x) \leqslant H^{k}\left(y,-D^{k} \varphi_{x}(y)\right)-H^{j}\left(x, D^{j} \varphi_{y}(x)\right) \\
= & H^{k}\left(y,-D^{k} \varphi_{x}(y)\right)-H^{k}\left(z,-D^{k} \varphi_{x}(y)\right) \\
& \quad+H^{j}\left(z, D^{j} \varphi_{y}(x)\right)-H^{j}\left(x, D^{j} \varphi_{y}(x)\right)+H^{k}\left(z,-D^{k} \varphi_{x}(y)\right)-H^{j}\left(z, D^{j} \varphi_{y}(x)\right) \\
& \leqslant \omega(d(y, z))+\omega(d(x, z))-H^{j}\left(z, D^{j} \varphi_{y}(x)\right)+H^{k}\left(z,-D^{k} \varphi_{x}(y)\right) .
\end{aligned}
$$

By (4.5), (4.6), (5.7) and (5.8) we get

$$
\begin{aligned}
H^{k}\left(z,-D^{k} \varphi_{x}(y)\right) & =\tilde{H}^{k}\left(\tilde{z},\left(-\partial_{1} \varphi_{x}(\tilde{y}), \ldots,-\partial_{n-1} \varphi_{x}(\tilde{y}),-\partial_{\nu_{k}} \varphi_{x}(\tilde{y})\right)\right. \\
& =\tilde{H}^{j}\left(\tilde{z},\left(\partial_{1} \varphi_{y}(\tilde{x}), \ldots, \partial_{n-1} \varphi_{y}(\tilde{x}),-\partial_{\nu_{j}} \varphi_{y}(\tilde{x})\right)\right. \\
& =\tilde{H}^{j}\left(\tilde{z},\left(\partial_{1} \varphi_{y}(\tilde{x}), \ldots, \partial_{n-1} \varphi_{y}(\tilde{x}), \partial_{\nu_{j}} \varphi_{y}(\tilde{x})\right)=H^{j}\left(z, D^{j} \varphi_{y}(x)\right) .\right.
\end{aligned}
$$

Hence

$$
\eta \leqslant-f(x) \leqslant \omega(d(y, z))+\omega(d(x, z))
$$

which gives a contradiction for $\epsilon \rightarrow 0^{+}$since by (5.4), $x, y \rightarrow x_{0}$ for $\epsilon \rightarrow 0$.

REMARK 5.1 For $H=\left(h^{j}(p)-f^{j}(x)\right)_{j \in J}$, with $h^{j}$ positively homogeneous, the comparison principle can be also obtained by means of Kružkov transform (see [4]).

\section{Existence}

In this section we prove existence of a solution via a Perron's method. For $u: \Omega \rightarrow \mathbb{R}$ we define $u^{*}, u_{*}: \Omega \rightarrow[-\infty, \infty]$ by

$$
u^{*}(x)=\limsup _{r \rightarrow 0}\{u(y): d(x, y) \leqslant r\} \quad \text { and } \quad u_{*}(x)=\liminf _{r \rightarrow 0}\{u(y): d(x, y) \leqslant r\} .
$$

LEMmA 6.1 Let $V$ be an arbitrary set of viscosity subsolutions of (4.7). Define $u(x):=$ $\sup _{v \in v} v(x)$ for all $x \in \Omega$ and assume that $u^{*}(x)<\infty$ in $\Omega$. Then $u^{*}$ is a viscosity subsolution of (4.7).

Proof. We assume that $x \in \Sigma$, otherwise we can use standard arguments in viscosity solution theory. We consider a $(j, k)$-upper test function $\varphi$ of $u^{*}$ at $x$ and we have to show that

$$
H^{j}\left(x, D^{j} \varphi(x)\right) \leqslant 0 .
$$

Let $\eta>0$ be such that $u^{*}-\varphi$ has a local maximum point at $x$ in $\bar{B}=B_{\eta}(x) \cap\left(\bar{\Omega}_{j} \cup \bar{\Omega}_{k}\right)$ where $B_{\eta}(x)=\{y \in \Omega: d(x, y) \leqslant \eta\}$. Since $\Omega$ is a LEP space we can assume that $\eta>0$ is sufficiently small such that $B_{\eta}(x) \cap \Sigma$ is contained in a $(n-1)$-dimensional affine linear subspace of $\mathbb{R}^{n+1}$. We consider the function $\varphi_{\delta}(y)=\varphi(y)+\delta d_{x}(y)^{2}$, for $\delta>0$, where $d_{x}(\cdot)=d(x, \cdot)$. Then $u^{*}-\varphi_{\delta}$ has a strict maximum point at $x$.

Let $\eta_{n} \rightarrow 0$ for $n \rightarrow \infty$ and $x_{n} \in B_{n}:=B_{\eta_{n}}(x)$ such that $\sup _{B_{n}} u-u\left(x_{n}\right) \leqslant 1 / n$. By the definition of $u$, there is $u_{n} \in V$ such that $u\left(x_{n}\right)-u_{n}\left(x_{n}\right) \leqslant 1 / n$. Hence

$$
\sup _{B_{n}} u \geqslant u_{n}\left(x_{n}\right)-\frac{1}{n}>\sup _{B_{n}} u-\frac{2}{n}
$$


and

$$
u^{*}(x)=\lim _{n} \sup _{B_{n}} u=\lim _{n} u_{n}\left(x_{n}\right) .
$$

Let $y_{n}$ be a maximum point for $u_{n}-\varphi_{\delta}$ in $\bar{B}$. Up to a subsequence, we can assume that $y_{n} \rightarrow z \in \bar{B}$. By Lemma 5.1 there exists a constant $C=C_{\bar{B}}$ such that

$$
u\left(y_{n}\right)-\varphi_{\delta}\left(y_{n}\right) \geqslant u_{n}\left(y_{n}\right)-\varphi_{\delta}\left(y_{n}\right) \geqslant u_{n}(x)-\varphi_{\delta}(x) \geqslant u_{n}\left(x_{n}\right)-\varphi_{\delta}\left(x_{n}\right)-C\left|x-x_{n}\right| .
$$

Taking the lim sup for $n \rightarrow \infty$ in the previous inequality, by (6.3) and the definition of lim sup* we get

$$
u^{*}(z)-\varphi_{\delta}(z) \geqslant u^{*}(x)-\varphi_{\delta}(x)
$$

and therefore $x=z$ since $u^{*}-\varphi_{\delta}$ has a strict maximum point at $x$. Moreover

$$
\lim _{n} y_{n}=x \quad \text { and } \quad \lim _{n} u_{n}\left(y_{n}\right)=u^{*}(x) .
$$

We distinguish two cases.

Case 1 . There are infinitely many $y_{n}$ with $y_{n} \notin \Sigma$. Then there exists a subsequence -also denoted with $\left\{y_{n}\right\}_{n}$ - which is completely contained in either $\Omega_{k}$ or $\Omega_{j}$. Without restriction we assume that $y_{n} \in \Omega_{k}$ for all $n$. Since $\varphi_{\delta}$ is an upper test function for $u_{n}$ at $y_{n}$ and $\varphi_{\delta}$ is differentiable at $y_{n}$, we get

$$
H^{k}\left(y_{n}, D^{k} \varphi_{\delta}\left(y_{n}\right)\right)=\tilde{H}^{k}\left(\tilde{y}_{n}, \partial^{k} \varphi\left(\tilde{y}_{n}\right)+2 d\left(x, y_{n}\right) \frac{\tilde{y}_{n}}{\left|\tilde{y}_{n}\right|}\right) \leqslant 0
$$

where $\tilde{y}_{n}=\mathfrak{i}_{x}\left(y_{n}\right)$ (recall that $\mathfrak{i}_{x}$ is the canonical identification around $x$ with $\mathfrak{i}_{x}(x)=0$ ).

Case 2. By possible truncating the sequence $\left\{y_{n}\right\}_{n}$ we can assume that $y_{n} \in \Sigma \cap \bar{B}$ for any $n$. Since $\Sigma$ is contained in a $(n-1)$-dimensional linear subspace of $\mathbb{R}^{n+1}$, we have

$$
\partial_{v_{j}} d_{x}(y)=0, \quad \forall y \in \Sigma \cap \bar{B}, j \in \operatorname{Inc}_{x} .
$$

In particular $d_{x}$ is $(j, k)$-differentiable in $\Sigma \cap \bar{B}$ and therefore $\varphi_{\delta}$ is $(j, k)$-differentiable in $\Sigma \cap B$ with $\partial_{m} \varphi_{\delta}(0)=\partial_{m} \varphi(0)$ for either $m=j$ or $m=k$. Since $\varphi_{\delta}$ is a $(j, k)$-upper test function for $u_{n}$ at $x$ we get

$$
H^{m}\left(y_{n}, D^{m} \varphi_{\delta}\left(y_{n}\right)\right)=\tilde{H}^{m}\left(\tilde{y}_{n}, \partial^{m} \varphi\left(\tilde{y}_{n}\right)\right) \leqslant 0
$$

for $\tilde{y}_{n}=\mathfrak{i}_{x}\left(y_{n}\right)$.

Now sending $n \rightarrow \infty$ and recalling (4.5) and (6.5), by (6.6) and (6.7) we get (6.1).

THEOREM 6.1 Assume that there is a viscosity subsolution $w \in U S C(\bar{\Omega})$ and a viscosity supersolution $W \in \operatorname{LSC}(\bar{\Omega})$ of (4.7) such that $w \leqslant W$ and

$$
w_{*}(x)=W^{*}(x)=g(x) \text { for } x \in \partial \Omega .
$$

Let the function $u: \bar{\Omega} \rightarrow \mathbb{R}$ be defined by $u(x):=\sup _{v \in X} v(x)$ where

$$
X=\{v \in \operatorname{USC}(\bar{\Omega}): v \text { is a viscosity subsolution of (4.7) with } w \leqslant v \leqslant W \text { on } \Omega\} .
$$

Then, $u^{*}$ and $u_{*}$ are respectively a subsolution and a supersolution to problem (4.7) in $\Omega$ with $u=g$ on $\partial \Omega$. 
Proof. We first show that $u$ is a subsolution of (4.7) with $u=g$ on $\partial \Omega$. Observe that

$$
g(x)=w_{*}(x) \leqslant u_{*}(x) \leqslant u(x) \leqslant u^{*}(x) \leqslant W^{*}(x)=g(x), \quad x \in \partial \Omega .
$$

Hence $u=u_{*}=u^{*}=g$ on $\partial \Omega$. Moreover, by Lemma $6.1, u^{*}$ is a viscosity subsolution of (4.7) in $\Omega$. Let us prove by contradiction that $u_{*}$ is a viscosity supersolution of (4.7) in $\Omega$ : we assume that $u_{*}$ does not satisfy the supersolution condition at some point $x_{0} \in \Omega$. We only consider the case $x_{0} \in \Sigma$, since the case $x_{0} \notin \Sigma$ is based upon similar, but easier, arguments. We assume that there exist a vector $p_{0} \in T_{x_{0}} \Sigma$, an index $j \in \operatorname{Inc}_{x_{0}}$ such that for any $k \in K:=\operatorname{Inc}_{x_{0}} \backslash\{j\}$, there exists a $(j, k)$-lower test function $\varphi_{k}$ of $u_{*}$ at $x_{0}$ with $\pi_{j}\left(D \varphi_{k}\left(x_{0}\right)\right)=p_{0}$ and

$$
H^{j}\left(x_{0}, D^{j} \varphi_{k}\left(x_{0}\right)\right)=H^{k}\left(x_{0}, D^{k} \varphi_{k}\left(x_{0}\right)\right)<0 .
$$

By adding a quadratic function as in the proof of Lemma 6.1, it is not restrictive to assume that $x_{0}$ is a strict minimum point for $u_{*}-\varphi_{k}$, for $k \in K$. Hence we can assume that $u_{*}\left(x_{0}\right)=\varphi_{k}\left(x_{0}\right)$ and there exists $\eta>0$ such that

$$
u_{*}(x)-\varphi_{k}(x)>0 \quad \text { for all } k \in K \text { and } x \in \partial B_{\eta}\left(x_{0}\right) \cap\left(\tilde{\Omega}_{j} \cup \tilde{\Omega}_{k}\right)
$$

where $B_{\eta}\left(x_{0}\right)=\left\{y \in \Omega: d\left(x_{0}, y\right)<\eta\right\}$. Moreover, by the continuity of $H$ and $D \varphi$,

$$
\begin{array}{ll}
H^{j}\left(x, D^{j} \varphi_{k}(x)\right)<0, & \forall x \in B_{j}:=B_{\eta}\left(x_{0}\right) \cap \tilde{\Omega}_{j}, \\
H^{k}\left(x, D^{k} \varphi_{k}(x)\right)<0, & \forall x \in B_{k}:=B_{\eta}\left(x_{0}\right) \cap \tilde{\Omega}_{k}, k \in K .
\end{array}
$$

Define

$$
\tilde{v}(z):= \begin{cases}\max _{k \in K} \varphi_{k}(z), & \text { if } z \in \Omega_{j}, \\ \varphi_{k}(z), & \text { if } z \in \Omega_{k}, K \neq J .\end{cases}
$$

Observe that since $\varphi_{k}$ are continuous on $\Sigma$ with $\varphi_{k}\left(x_{0}\right)=u_{*}\left(x_{0}\right)$ and $\pi_{j}\left(D^{j} \varphi_{k}\left(x_{0}\right)\right)=p_{0}$ for any $k \in K$, then the function $\tilde{v}$ is continuous $B_{\eta}\left(x_{0}\right)$. We claim that $\tilde{v}$ is a subsolution of (4.7) in $B_{\eta}\left(x_{0}\right)$.

Case 1: Consider $x \in B_{\eta}\left(x_{0}\right) \cap \Sigma$. If $\psi$ is a $(l, m)$-upper test function to $\tilde{v}$ at $x$ for $l, m \in \operatorname{Inc}_{x}$, $l \neq m$, we have to show that

$$
H^{l}\left(x, D^{l} \psi(x)\right)=\tilde{H}^{l}\left(\tilde{x}, \partial^{l} \psi(\tilde{x})\right) \leqslant 0
$$

where $\tilde{x}=\mathfrak{i}_{x_{0}}(x)$. Consider first the case $l, m \neq j$. By the definition of $\tilde{v}$ it follows that

$$
\partial_{i} \psi(x)=\partial_{i} \varphi_{m}(x) \quad \text { for all } i=, 1 \ldots, n-1 \quad \text { and } \quad \partial_{v_{m}} \psi(x) \geqslant \partial_{v_{m}} \varphi_{m}(x) .
$$

Hence

$$
\left|\partial_{v_{m}} \psi(x)\right| \leqslant\left|\partial_{v_{m}} \varphi_{m}(x)\right| \text { provided that } \partial_{v_{m}} \psi(x) \leqslant 0 .
$$

Applying a similar argument we get that

$$
\left|\partial_{v_{l}} \psi(x)\right| \leqslant\left|\partial_{v_{l}} \varphi_{m}(x)\right| \text { provided that } \partial_{v_{l}} \psi(x) \leqslant 0 .
$$

By (6.14), (6.15) and since $\psi$ is $(l, m)$-differentiable at $x$, we have

$$
\left|\partial_{v_{l}} \psi(x)\right|=\left|\partial_{v_{m}} \psi(x)\right| \leqslant \max \left\{\left|\partial_{v_{l}} \varphi_{l}(x)\right|,\left|\partial_{v_{m}} \varphi_{m}(x)\right|\right\} .
$$


By the assumptions (4.2)-(4.6), for $s \in \operatorname{Inc}_{x}$, the function $h: \mathbb{R} \rightarrow \mathbb{R}$ defined by

$$
p_{n} \mapsto h\left(p_{n}\right):=H^{s}\left(\tilde{x},\left(\partial_{1} \psi(x), \ldots, \partial_{n-1} \psi(x), p_{n}\right)\right)
$$

is independent of $s$, symmetric at $p_{n}=0$, and increasing in $\left|p_{n}\right|$. Hence by (6.12), (6.16)

$$
\begin{aligned}
H^{l}\left(x, D^{l} \psi(x)\right) & =\tilde{H}^{l}\left(\tilde{x}, \partial^{l} \psi(x)\right)=h\left(\partial_{v_{l}} \psi(x)\right) \leqslant \max \left\{h\left(\partial_{\nu_{l}} \varphi_{l}(x)\right), h\left(\partial_{v_{m}} \varphi_{m}(x)\right)\right\} \\
& =\max \left\{\tilde{H}^{l}\left(x, \partial^{l} \varphi_{l}(x)\right), \tilde{H}^{m}\left(x, \partial^{m} \tilde{\varphi}_{m}(x)\right)\right\} \\
& =\max \left\{H^{l}\left(x, D^{l} \varphi_{l}(x)\right), H^{m}\left(x, D^{m} \varphi_{m}(x)\right)\right\}<0
\end{aligned}
$$

and we obtain (6.13).

We now consider the case where one of the indices $l, m$ coincides with $j$, i.e., $\psi$ is a $(j, m)$-upper test function of $\tilde{v}$ at $x$. As above we get that

$$
\begin{array}{r}
\partial_{i} \psi(x)=\partial_{i} \varphi_{k}(x) \text { for } i=, 1 \ldots, n-1, k \in K, \\
\partial_{\nu_{j}} \psi(x) \geqslant \max \left\{\max _{k \in K} \partial_{\nu_{j}} \varphi_{k}(x), \partial_{v_{m}} \varphi_{m}(x)\right\}
\end{array}
$$

and therefore

$$
\left|\partial_{\nu_{j}} \psi(x)\right|=\left|\partial_{v_{m}} \psi(x)\right| \leqslant \max \left\{\max _{k \in K}\left|\partial_{\nu_{j}} \varphi_{k}(x)\right|,\left|\partial_{\nu_{m}} \varphi_{m}(x)\right|\right\}
$$

Hence we proceed as above to show the subsolution condition (6.13).

Case 2: Let $x \in B_{\eta}\left(x_{0}\right) \backslash \Sigma$. If $x \in \Omega_{l}, l \neq j$, then $\tilde{v}(x)=\varphi_{l}(x)$. Since $\varphi_{l}$ is differentiable at $x$, we get immediately the subsolution condition by (6.12). If $l=j$, then by (6.12), we have

$$
H^{k}\left(x, D^{k} \varphi_{k}(x)\right)<0, \quad \text { for any } k \in K
$$

and the claim follows by Lemma 6.1.

We set $\tilde{v}_{\epsilon}=\tilde{v}+\epsilon$. Then $\tilde{v}_{\epsilon}$ is still a subsolution and by (6.11), for $\epsilon$ sufficiently small, $\tilde{v}_{\epsilon}(x)<$ $u_{*}(x)$ for $x \in \partial B_{\eta}\left(x_{0}\right)$. Since $u_{*} \leqslant u$ on $\Omega$ we have $\tilde{v}_{\epsilon}<u$ on $\partial B_{\eta}\left(x_{0}\right)$. Hence the function $v: \Omega \rightarrow \mathbb{R}$ given by

$$
v(x)= \begin{cases}\max \left\{u(x), \tilde{v}_{\epsilon}(x)\right\}, & \text { if } x \in B_{\eta}\left(x_{0}\right) \\ u(x), & \text { if } x \notin B_{\eta}\left(x_{0}\right)\end{cases}
$$

is upper semi-continuous in $\Omega$ and, by Lemma 6.1, it is a subsolution of (4.7). It follows that $v \in X$. Since $v\left(x_{0}\right)=u_{*}\left(x_{0}\right)+\epsilon$, there is $\bar{x} \in B_{\eta}\left(x_{0}\right)$ such that $v(\bar{x})>u(\bar{x})$ and we get a contradiction to the definition of $u$.

The proof of the next proposition is based on arguments similar to those used for Lemma 6.1 and Theorem 6.1 (see also [23, prop.3.5])

Proposition 6.1 Let $v$ be an arbitrary set of solutions of (4.7). Define $u(x):=\inf _{v \in v} v(x)$ for all $x \in \Omega$ and assume that $u(x) \in \mathbb{R}$ for some $x \in \Omega$. Then $u$ is a solution of (4.7). 


\section{A representation formula for the solution of the Dirichlet problem}

In this section, we shall assume that, beside hypotheses (4.2)-(4.6), $H$ also fulfills

$$
H^{j}(x, p) \text { is strictly convex in } p \in \mathbb{R}^{n} \text {, for } x \in \tilde{\Omega}_{j} .
$$

Observe that, under assumptions (7.1) and (4.6), hypothesis (4.4) becomes redundant (see also remark 4.1 for others comments on the hypotheses). Our aim is to introduce a distance-like function and to give a representation formula for the solution of the Dirichlet problem.

Definition 7.1 Let $x, y \in \bar{\Omega}$ and $T>0$. A continuous curve $\gamma:[0, T] \rightarrow \bar{\Omega}$ is called a connection of $x$ and $y$ if

- $\gamma(0)=x, \gamma(T)=y$;

- $\gamma([0, T]) \subset \bar{\Omega}$

- $\gamma$ is an absolutely continuous path in the sense that there are $t_{0}:=0<t_{1}<\cdots<t_{M+1}:=T$ such that for any $m=0, \ldots, M$, we have $\gamma\left(\left[t_{m}, t_{m+1}\right]\right) \subset \bar{\Omega}_{j_{m}} \subset \mathbb{R}^{n}$ for some $j_{m} \in J$ and the curve $\gamma_{m}:\left[t_{m}, t_{m+1}\right] \rightarrow \bar{\Omega}_{j_{m}}$ defined by $\gamma_{m}(t):=\gamma(t)$ is absolutely continuous. We assume that the number $t_{m}$ is maximal with the property that $\gamma\left(\left[t_{m}, t_{m+1}\right]\right) \subset \bar{\Omega}_{j_{m}}, m=1, \ldots, M$.

We denote by $\mathbb{B}_{x, y}^{T}$ the set of all connections of $x$ and $y$. For $\gamma \in \mathbb{B}_{x, y}^{T}$, we define the length by $\mathcal{L}(\gamma):=\sum_{l=1}^{M} \mathcal{L}\left(\gamma_{l}\right)$ where $\mathcal{L}\left(\gamma_{l}\right)$ is the usual length in $\mathbb{R}^{n}$.

For $x, y \in \Omega$, we define

$$
S(x, y)=\inf \left\{\int_{0}^{T} L(\gamma(s), \dot{\gamma}(s)) d s: T>0, \gamma \in \mathbb{B}_{x, y}^{T}\right\},
$$

where $\mathbb{B}_{x, y}^{T}$ as in Definition 7.1 and $L=\left(L^{j}\right)_{j \in J}$ is a family of mappings $L^{j}: \tilde{\Omega}_{j} \times T_{x}^{j} \Omega \rightarrow \mathbb{R}$ defined by

$$
L^{j}(x, q):=\sup _{p \in \mathbb{R}^{n}}\left\{p \cdot q-H^{j}(x, p)\right\}, \quad x \in \Omega_{j}, q \in \mathbb{R}^{n} .
$$

Proposition 7.1 Assume that there exists a subsolution of (4.7). Then

(i) For any $x, y \in \Omega, S(x, y)$ is finite, Lipschitz continuous in its variables, $S(x, x)=0$ and satisfies the triangular inequality. Moreover for any subsolution $u$ of (4.7)

$$
u(x)-u(y) \leqslant S(y, x), \quad \text { for any } x, y \in \Omega
$$

(ii) For any fixed $x \in \Omega, u(\cdot)=S(x, \cdot)$ is a subsolution of (4.7) in $\Omega$ and a supersolution in $\Omega \backslash\{x\}$.

Proof. The proof of (i) is standard and for details we refer to [14]. For (ii), we first prove that $u$ is a subsolution in $\Omega$. We consider $y \in \Omega$ and we distinguish two cases.

Case 1: $y \in \Omega_{j}$ for some $j \in J$. We recall that, near $y, \Omega$ is locally homeomorphic to a $n$ dimensional Euclidean space via the canonical identification chart $\left(V_{y}, \mathfrak{i}_{y}\right)$ (see prop. 3.1). Recall also that $\mathfrak{i}_{y}(y)=0$. For $q \in \mathbb{R}^{n}$, we consider $t>0$ sufficiently small in such a way that $\tilde{z}_{t}:=$ $t q \in \mathfrak{i}_{y}\left(V_{y}\right)$ and set $z_{t}=\mathfrak{i}_{y}^{-1}(t q) \in V_{y}$. Define a trajectory $\gamma \in \mathbb{B}_{z t, y}^{t}$ by $\gamma_{t}(s)=\mathfrak{i}_{y}^{-1}\left((1-s / t) \tilde{z}_{t}\right)$, $s \in[0, t]$. Let $\psi$ be an upper test function for $u(\cdot)=S(x, \cdot)$ at $y$, hence $u(y)-\psi(y) \geqslant u(z)-\psi(z)$ for $z \in V_{y}$. We want to show that

$$
H^{j}\left(y, D^{j} \psi(y)\right) \leqslant 0 .
$$


We have

$$
\begin{aligned}
\partial^{j} \psi(0) \cdot q & =\partial^{j}\left(\psi \circ \mathfrak{i}_{y}^{-1}\right)(0) \cdot q=\lim _{t \rightarrow 0^{+}} \frac{\psi\left(\mathfrak{i}_{y}^{-1}\left(\tilde{z}_{t}\right)\right)-\psi\left(\mathfrak{i}_{y}^{-1}(0)\right)}{t} \\
& \leqslant \lim _{t \rightarrow 0^{+}} \frac{S\left(x, z_{t}\right)-S(x, y)}{t} \leqslant \lim _{t \rightarrow 0^{+}} \frac{S\left(z_{t}, y\right)}{t} \leqslant \lim _{t \rightarrow 0^{+}} \frac{1}{t} \int_{0}^{t} L^{j}\left(\gamma_{t}(s), \dot{\gamma}_{t}(s)\right) d s \\
& =\lim _{t \rightarrow 0^{+}} \frac{1}{t} \int_{0}^{t} L^{j}\left(\mathfrak{i}_{y}^{-1}\left((1-s / t) \tilde{z}_{t}\right), q\right) d s=\tilde{L}(0, q)
\end{aligned}
$$

where $\tilde{L}^{j}(y, q)=L^{j}\left(\mathfrak{i}_{y}^{-1}(y), q\right)$ is the dual function of the Hamiltonian $\tilde{H}^{j}(y, p)$ defined in (4.1). By the previous inequality and the arbitrariness of $q$ we get $\tilde{H}^{j}\left(0, \partial^{j} \psi(0)\right)=\sup _{q \in \mathbb{R}^{n}}\left\{\partial^{j} \psi(0)\right.$. $\left.q-\tilde{L}^{j}(0, q)\right\} \leqslant 0$ and therefore (7.4) thanks to (4.1).

Case 2: $y \in \Sigma$. In this case, near $y, \Omega$ is locally homeomorphic to an elementary ramified space $\mathbb{R}_{r(y)}^{n}$. As before we consider the canonical immersion $\left(V_{y}, \mathfrak{i}_{y}\right)$. Let $\psi$ be an $(j, k)$-upper test function to $u(\cdot)=S(x, \cdot)$ at $y$. For $q \in \mathfrak{i}_{y}\left(\tilde{\Omega}_{j} \cup \tilde{\Omega}_{k}\right)$, we consider $z_{t}$ and $\tilde{z}_{t}$ as before and we introduce the trajectory $\gamma \in \mathbb{B}_{z_{t}, y}^{t}$ by $\gamma_{t}(s)=\mathfrak{i}_{y}^{-1}\left((1-s / t) \tilde{z}_{t}\right), s \in[0, t]$. We have two cases

$$
\begin{aligned}
& \gamma_{t}(s) \in \Sigma \cap V_{y} \text { for any } s \in[0, t], \\
& \gamma_{t}(s) \in \Omega_{m} \cap V_{y} \text { for any } s \in[0, t] \quad \text { for either } m=j \text { or } m=k .
\end{aligned}
$$

If (7.5) is satisfied, since $\gamma_{t} \in \tilde{\Omega}_{j} \cap \tilde{\Omega}_{k}$, arguing as above we get that

$$
\partial^{m} \psi(0) \cdot q-\tilde{L}^{m}(0, q) \leqslant 0 \quad \text { for } m=k, j .
$$

In other case, we get

$$
\partial^{m} \psi(0) \cdot q-\tilde{L}^{m}(0, q) \leqslant 0 \quad \text { for either } m=j \text { or } m=k .
$$

If $m=k$, since $\psi$ is differentiable and (4.5) holds, we have

$$
\partial^{k} \psi(0) \cdot q-\tilde{L}^{k}(0, q) \leqslant 0=\left(\partial_{1} \psi(0), \ldots, \partial_{n-1} \psi(0),-\partial_{\nu_{j}} \psi(0)\right) \cdot q-\tilde{L}^{j}(0, q) \leqslant 0
$$

Recalling (4.6), by (7.7) and (7.8), we get (7.4).

The proof that $u$ is a supersolution is based on the same argument of the proof of theorem 6.1. In fact, by (7.3) and the part already proved, it follows immediately that

$$
u(y)=\max \{v(y): v \text { is a subsolution of (4.7) s.t. } v(x)=0\} .
$$

If we assume by contradiction that $u$ is not a supersolution at some point $z \in \Omega \backslash\{y\}$, we get a contradiction to (7.9) showing as in the proof of Theorem 6.1 that there exists a subsolution $v$ of (4.7) such that $v(z)>u(z)$.

In the following theorem, we give a representation formula for the solution of the Dirichlet problem for (4.7).

THEOREM 7.1 Assume that there exists a differentiable function $\psi$ and a continuous function $h$ with $h<0$ such that $H(x, D \psi) \leqslant h(x)$ for $x \in \Omega$. 
Consider the Hamilton-Jacobi equation (4.7) with the boundary condition

$$
u(x)=g(x), \quad x \in \partial \Omega
$$

where $g: \partial \Omega \rightarrow \mathbb{R}$ is a continuous function satisfying

$$
g(x)-g(y) \leqslant S(y, x) \quad \text { for any } x, y \in \partial \Omega .
$$

Then the unique viscosity solution of (4.7)-(7.11) is given by

$$
u(x):=\min \{g(y)+S(y, x): y \in \partial \Omega\} .
$$

Proof. First observe that if $u(x) \neq g(x)$ for some $x \in \partial \Omega$, then there is $z \in \partial \Omega$ such that $g(x)>S(z, x)+g(z)$, which gives a contradiction to (7.12). Hence $u=g$ on $\partial \Omega$.

By Proposition 6.1 and Proposition 7.1.(ii), $u$ is a solution of (4.7). To show that it is the unique solution, assume that there exists another solution $v$ of (4.7)-(7.11). For $\theta \in(0,1)$ define $u_{\theta}:=$ $\theta u+(1-\theta) \psi$, where $\psi$ as in (7.10). By adding a constant it is not restrictive to assume that $\psi$ is sufficiently small in such a way that

$$
u_{\theta}(x) \leqslant u(x), \quad x \in \Omega .
$$

If $x \in \Omega_{j}$ and $\varphi$ is an upper test function of $u$ at $x$, we set $\varphi_{\theta}:=\theta \varphi+(1-\theta) \psi$ and we obtain by means of convexity of $H$

$$
H^{j}\left(x, D^{j} \varphi_{\theta}\right) \leqslant \theta H^{j}\left(x, D^{j} \varphi\right)+(1-\theta) H^{j}\left(x, D^{j} \psi\right) \leqslant(1-\theta) h(x) .
$$

If $x \in \Sigma$ and $\varphi$ is an $(j, k)$-upper test function of $u$ at $x$, then $\varphi_{\theta}:=\theta \varphi+(1-\theta) \psi$ is a $(j, k)$-upper test function of $u_{\theta}$ at $x$ and we again obtain (7.14). Hence $u_{\theta}$ is a viscosity subsolution of

$$
H\left(x, D^{j} u\right) \leqslant(1-\theta) h(x) .
$$

Applying Theorem 5.1 with $f=(1-\theta) h$ and (7.13), we get $u_{\theta} \leqslant v$ for all $\theta \in(0,1)$. Letting $\theta$ tend to 1 yields $u \leqslant v$. Exchanging the role of $u$ and $v$ we conclude that $u=v$ in $\Omega$.

REMARK 7.1 The Assumption (7.10) is satisfied if $H(x, 0)<0$ for any $x \in \Omega$ by taking $\psi \equiv$ $c \in \mathbb{R}$. If $f>0$ in $\Omega$ then the Hamiltonian $H=\left(H_{j}\right)_{j \in J}$, where $H^{j}(x, p)=|p|^{2}-f^{j}(x)$ (see Example 4.1), satisfies this assumption.

\section{REFERENCES}

1. Achdou, Y., Camilli, F., Cutrì, A. And Tchou, N., Hamilton-Jacobi equations constrained on networks, to appear in NoDEA Nonlinear Differential Equations Appl. (available on http://hal. archives-ouvertes.fr).

2. Alvarez, O. AND BARdi, M., Ergodicity, stabilization, and singular perturbations for Bellman-Isaacs equations, Mem. Amer. Math. Soc. 204 (2010), Zbl1209. 35001 MR2640736

3. Azagra, D., Ferrera, J. And López-Mesas, F., Nonsmooth analysis and Hamilton-Jacobi equations on Riemannian manifolds. J. Funct. Anal. 220 (2005), 304-361. Zbl1067. 49010 MR2119282

4. Bardi, M. And CApuzzo Dolcetta, I., Optimal control and viscosity solutions of Hamilton-JacobiBellman equations. Birkhäuser Boston Inc., Boston, MA, 1997. Zb10890 . 49011 MR1484411 
5. Barles, G., Briani, A. And Chasseigne, E., A Bellman approach for two-domains optimal control problems in $\mathbb{R}^{N}$, preprint (avaliable on http: //arxiv.org/abs/1112.3727).

6. Bressan, A. And Hong, Y., Optimal control problems on stratified domains, Netw. Heterog. Media 2 (2007), 313-331. Zbl1123.49028 MR2291823

7. Camilli, F., Festa, A. And Schieborn, D., An approximation scheme for an Hamilton-Jacobi equation defined on a network, arXiv:1105.5725 (2011).

8. Cannarsa, P. and Cardaliaguet, P., Representation of equilibrium solutions to the table problem for growing sandpiles, J. Eur. Math. Soc. (JEMS) 6 (2004), 435-464. Zbl1084. 35015 MR2094399

9. Cardaliaguet, P., Nolen, J. and Souganidis, P., Homogenization and enhancement for the Gequation, Arch. Ration. Mech. Anal. 199 (2011), 527-561. Zblpre05952948 MR2763033

10. FAthi, A. And SiCONOLFI, A., Existence of $C^{1}$ critical subsolutions of the Hamilton-Jacobi equation, Invent. Math. 155 (2004), 363-388. Zbl1061.58008 MR2031431

11. Freidlin, M.I. AND Wentzell, A.D., Diffusion processes on an open book and the averaging principle, Stochastic Process. Appl. 113 (2004), 101-126. Zbl1075.60100 MR2078539

12. GigA, Y., Surface evolution equations. A level set approach. Monographs in Mathematics, 99. Birkhäuser Verlag, Basel, 2006. Zbl1096.53039 MR2238463

13. Kogut, P.I. And Leugering, G., Homogenization of constrained optimal control problems for onedimensional elliptic equations on periodic graphs, ESAIM Control Optim. Calc. Var. 15 (2009), 471-498. Zbl1173.35015 MR2513095

14. IsHII, H., Asymptotic solutions for large time of Hamilton-Jacobi equations in Euclidean $n$ space. Ann. Inst. H. Poincaré Anal. Non Linéaire 25 (2008), 231-266. Zbl1145. 35035 MR2396521

15. Imbert, C., Monneau, R. And Zidani, H., A Hamilton-Jacobi approach to junction problems and application to traffic flows, ESAIM, Control Optim. Calc. Var. 19 (2013), 129-166. Zblpre06144570

16. Lagnese, J.E., Leugering, G. And SchmidT, E.J.P.G., Modeling, analysis and control of dynamic elastic multi-link structures. Systems \& Control: Foundations \& Applications. Birkhäuser (1994). Zb10810.73004 MR1279380

17. Lagnese, J.E. And Leugering, G., Domain decomposition methods in optimal control of partial differential equations. International Series of Numerical Mathematics 148. Birkhäuser (2004). Zbl1059. 49002 MR2093789

18. Lumer, G., Espaces ramifiés, et diffusions sur les réseaux topologiques, C. R. Acad. Sci. Paris Sér. A-B 291 (1980), A627-A630. Zb10449.35110 MR0606449

19. Mantegazza, C. And Mennucci, A., Hamilton-Jacobi equations and distance functions on Riemannian manifolds, Appl. Math. Optim. 47 (2003), 1-25. Zbl1048. 49021 MR1941909

20. NiCAISE, S., Elliptic operators on elementary ramified spaces, Integral Equations and Operator Theory 11 (1988), 230-257. Zbl0649.35027 MR0928484

21. NiCAISE, S., Polygonal interface problems. Methoden und Verfahren der Mathematischen Physik, volume 39. Verlag Peter D. Lang (1993). Zbl0794. 35040 MR1236228

22. SCHIEBORN, D., Viscosity solutions of Hamilton-Jacobi equations of Eikonal type on Ramified Spaces. Ph.D. thesis, Tübingen (2006).

23. Schieborn, D. And CAmilli, F., Viscosity solutions of Eikonal equations on topological network, Calc. Var. Partial Differential Equations 46 (2013), 671-686. Zblpre06145935 MR3018167

24. Soravia, P., Generalized motion of front along its normal direction: a differential game approach, Nonlinear Analysis TMA 22 (1994), 1247-1262. Zbl0814. 35140 MR1279982

25. Williams, R. F., One-dimensional non-wandering sets, Topology 6 (1967), 473-487. Zb10159. 53702 MR0217808 